\title{
Periférico e comunitário: características e desafios de um programa de pós-graduação em Psicologia no Centro-Oeste
}

\author{
Daniela Sacramento Zanini. Pontifícia Universidade Católica de Goiás \\ Margareth Regina Gomes Verissimo Faria. Pontifícia Universidade Católica de Goiás \\ Lauro Eugênio Guimarães Nalini. Pontifícia Universidade Católica de Goiás \\ Cristiano Coelho. Pontifícia Universidade Católica de Goiás \\ Helenides Mendonça. Pontifícia Universidade de Lisboa \\ Sebastião Benicio da Costa Neto. Pontifícia Universidade Católica de Goiás \\ Iorhana Almeida Fernandes. Centro Universitário de Anápolis/GO \\ Lila Maria Spadoni Lemes. Pontifícia Universidade Católica de Goiás
}

\section{Resumo}

Este estudo tem como objetivos avaliar em caráter descritivo e exploratório, associações entre investimento externo e produção docente e identificar os eixos temáticos das teses e dissertações produzidas nas duas últimas décadas no Programa de Pósgraduação em Psicologia da PUC Goiás. Foram analisados os dados do coleta Capes e da Plataforma Sucupira para avaliar a correlação entre investimento e produção científica, além de 153 dissertações e 19 teses, selecionadas por amostragem sistemática e analisadas com o apoio do Iramuteq, de maneira a identificar os eixos temáticos dos trabalhos desenvolvidos. Os dados indicam correlação entre investimento financeiro e produção científica e originaram os seguintes eixos temáticos: análise e evolução do comportamento; psicologia social, trabalho e organizações; psicopatologia clínica e psicologia da saúde. A produção científica analisada abrange desde aspectos individuais a temáticas ligadas às relações intra e intergrupais. Ademais, os resultados demonstram que os recursos externos e a maturidade acadêmica tendem a aumentar concomitantemente.

Palavras-chave: desenvolvimento social; formação profissional; história da psicologia; pós-graduação; produção científica.

\section{Abstract}

Peripheral and communitarian: Characteristics and challenges of a Midwest graduate program in Psychology. This study aims a descriptive and exploratory evaluation of the association between external investment and scientific production and to identify the thematic axes of thesis and dissertations produced in the last two decades in the Post-Graduation Program in Psychology of PUC Goiás. Data from the Coleta CAPES and the Sucupira platform were analyzed to evaluate the relation between investment and scientific production and 153 dissertations and 19 theses selected by systematic sampling and analyzed with the support of Iramuteq to identify the thematic axes of the works developed. The data indicate the association between financial investment and scientific production and identify the following thematic axes: analysis and evolution of behavior; social psychology, work and organizations; clinical psychopathology and health psychology. The analyzed scientific production ranges from individual aspects to topics related to intra and intergroup relations. In addition, the results demonstrate that external resources and the program academic maturity increase_concomitantly.

Keywords: social development; professional qualification; history of psychology; postgraduate studies; scientific production.

\section{Resumen}

Periférico y comunitario: características y desafíos de un programa de posgrado en Psicología en el Centro-Oeste. Este estudio tiene como objetivos evaluación decriptiva e exploratória de las asociaciones entre inversión externa y producción docente e identificar los ejes temáticos de las tesis y disertaciones producidas en las dos últimas décadas en el Programa de Postgrado en Psicologia de la PUC Goiás. Se analizaron los datos del Coleta Capes y de la Plataforma Sucupira para evaluar la relación entre inversión y producción científica, así como 153 disertaciones y 19 tesis, seleccionadas por muestreo sistemático y analizadas con el apoyo del Iramuteq, para identificar los ejes temáticos de los trabajos desarrollados. Los datos indican asociacion entre inversión financiera y producción científica e identificaron los siguientes ejes temáticos: análisis y evolución del comportamiento; psicología social, trabajo y organizaciones; psicopatología clínica y psicología de la salud. La producción científica analizada abarca desde aspectos individuales a temáticas ligadas a las relaciones intra e intergrupales. Además, los resultados demuestran que los aumentos de recursos externos y de_la madurez académica del programa son concomitantes.

Palabras clave: desarrollo social; formación profesional; historia de la psicología; posgraduación; producción científica. 
O processo de interiorização dos Programas de Pós-Graduação em Psicologia (PPGP) é um fenômeno complexo, relacionado à expansão do trabalho de psicólogos fora dos principais centros urbanos brasileiros (Bastos, Gondim, \& Rodrigues, 2010; Macedo \& Dimenstein, 2011). A interiorização da pós-graduação tem se estabelecido com dois propósitos: a busca de qualificação profissional, incluindo a necessidade de compreensão de distintas realidades pessoais, sociais e institucionais (seja por profissionais da psicologia, seja por outros profissionais de distintas áreas); e o redirecionamento de recursos financeiros para a formação de pesquisadores que antes tinham as instituições de ensino no exterior como únicas alternativas (Tourinho \& Bastos, 2010). A qualificação profissional inclui a necessidade de compreensão de distintas realidades pessoais, sociais e institucionais, seja por profissionais da psicologia, sou por outros profissionais de outras áreas.

Esse processo de interiorização está diretamente relacionado à própria expansão da pós-graduação brasileira, que, de acordo com Costa e Yamamoto (2016), é uma modalidade educacional exitosa, baseada em um "metódico sistema de avaliação institucional, do investimento contínuo por parte do poder público e da mobilização permanente da comunidade acadêmico-científica nacional" (p. 457).

Macedo e Dimenstein (2011) evidenciaram que a interiorização e a descentralização da formação em psicologia para além dos grandes centros urbanos tinha como explicação a existência de políticas públicas que levaram à reestruturação de serviços voltados ao bem-estar nas comunidades, a reorganização e a expansão da educação superior e as mudanças nos processos de reestruturação urbana no Brasil. Tal processo de interiorização e descentralização do ensino superior teve relevante contribuição das Instituições Comunitárias de Ensino Superior (ICES) que, segundo Venanzi e Sandano (2014), tinham por vocação as ações de ensino, pesquisa e extensão, com foco nas comunidades locais e regionais.

Veiga (2014), ao comparar as características do ensino e da investigação nas áreas da psicologia e da educação nos contextos brasileiro e português apontou que quanto maior a escolarização do indivíduo, menor é a importância dada por ele a valores como justiça e solidariedade. Para o autor isso se reflete em uma produção científica menos comprometida com os problemas atuais da sociedade e mais reativa a uma lógica produtivista científica. Apesar disso, observa-se que a Coordenação de Aperfeiçoamento de Pessoal de Nível Superior (CAPES, 2017), e particularmente no contexto da avaliação dos programas de pós-graduação stricto sensu em psicologia, tem buscado minimizar essa lógica produtivista por meio da busca de uma maior qualidade da formação e da produção científica. Neste sentido, cinco quesitos são considerados, quais sejam: a proposta do programa; o corpo docente; o corpo discente, as teses e dissertações; a produção intelectual; e, a inserção social.

No contexto da produção científica, as ICES têm por característica se posicionar entre as instituições públicas e as privadas de ensino, se destacando por sua responsabilidade social, demarcada pela proposição de avanço na formação humana e na identificação e problematização de demandas produzidas no e para o contexto social (Venanzi \& Sandano, 2014), sem afastar-se daquilo que se debate no plano internacional e multicultural. Assim, desde o início deste século, as ICES eram reconhecidas, também, por sua participação na pós-graduação brasileira, em número de programas de pós-graduação stricto sensu (PPGSS) que perdia apenas para as instituições públicas de ensino. Contudo, a distribuição desses PPGSS de ICES ocorreu de forma heterogênea no país (Morosini \& Franco, 2006; Steiner, 2005).

No Centro-Oeste brasileiro, excetuando o Distrito Federal, tanto os cursos de graduação quanto os de Pós-Graduação Stricto Sensu em Psicologia (PPGP) foram propostos por ICES, num período em que eram únicos na região: na Universidade Católica Dom Bosco (UCDB), antes Faculdades Unidas Católicas de Mato Grosso (FUCMT), em Campo Grande, com a graduação criada em 1975 (Delmondes, 2018) e o PPGP em 1997; e, na Pontifícia Universidade Católica de Goiás - antes Universidade Católica de Goiás -, em Goiânia, com a graduação em Psicologia criada em 1973 e o PPGP em 1999. Tais programas tiveram um protagonismo ao captar a demanda para a formação de pesquisadores e professores para o ensino superior, muitos deles vinculados a instituições com distintas características, como IES não comunitárias e com fins lucrativos, provenientes de regiões distantes e sem oferta de pós-graduação na área.

Particularmente, o PPGP da Pontifícia Universidade Católica de Goiás (PUC Goiás), apesar do foco para o estudo de objetos próprios e à luz da psicologia, teve um corpo de discentes constituído por profissionais de diferentes áreas do conhecimento. Isso Ihe proporcionou uma aproximação multidimensional e complexa de temas, cujas características vinham das indagações do mundo real e institucional do trabalho ligadas às linhas

Estudos de Psicologia, 24(2), abril a junho de 2019, 113-124 
de investigação, ainda que, também, acolhessem múltiplas demandas das pesquisas básica e teórico-conceitual. O impacto social do PPGP da PUC Goiás na sociedade foi evidenciado com a efetivação de muitos de seus egressos na educação superior como docentes e/ou em outras funções institucionais, em diversos municípios das regiões Centro-Oeste e Norte do País.

O PPGP da PUC Goiás, após o último quadriênio de avaliação da Coordenação de Aperfeiçoamento de Pessoal de Nível Superior - Capes (2013-2016), tem passado por mudanças em sua proposta formativa, incluindo a revisão de suas linhas de pesquisa. Assim, desde 2018, as linhas de pesquisa já se organizam em torno de três eixos que ancoram pontos centrais dos diferentes projetos e produções, a saber: Análise do Comportamento; Saúde - Processos, Avaliação e Intervenção; e Processos Psicossociais (http://sites.pucgoias.edu.br/pos-graduacao/mestrado-doutorado-psicologia). Tais eixos conseguem acomodar grande parte dos interesses dos discentes que recorrem à instituição para sua formação em nível de mestrado e doutorado.

As IES Comunitárias, por sua própria vocação e responsabilidade no processo de interiorização da pós-graduação stricto sensu brasileira, cumprem um papel social fundamental na formação qualificada, somando-se aos esforços da Capes para responder às fortes demandas nacionais por educação superior neste país de dimensões territoriais continentais. Nesse sentido, tomando como questões norteadoras a delimitação do perfil da produção acadêmica do PPGP da PUC Goiás e os desafios dos PPGs de Psicologia fora do eixo sul-sudeste, este estudo tem como objetivo avaliar o impacto do investimento externo (bolsas e recursos advindos de projetos financiados) na produção docente assim como identificar os focos temáticos centrais das teses e dissertações produzidas nas duas últimas décadas.

A relevância dessa relação é abordada a partir da consideração de que a interiorização de um programa envolve lidar com as demandas próprias dos contextos nos quais se insere e imprime desafios ao desenvolvimento de uma cultura de pesquisa e formação avançada em psicologia. Nesse sentido, pretende-se ponderar como o aumento do financiamento e a maturidade do programa contribuíram para a produção voltada para questões regionais relevantes, as quais poderiam ser negligenciadas ou potencialmente subestimadas não fosse o processo de interiorização ao qual a pós-graduação foi submetido. De acordo com o Plano Nacional de Pós-Graduação (PNPG) 2011-2010 (Capes, 2010), a expansão da pós-graduação no Brasil no período anterior (2005-2010) também foi acompanhada de um processo de interiorização. Nesse processo, o CentroOeste, que congregava $5,48 \%$ dos cursos de mestrado e doutorado em 2004, passou para 6,89\%, em 2010, e, atualmente, ${ }^{1}$ conta com $8,37 \%$. O crescimento dessa porcentagem envolveu tanto a expansão das Instituições Federais de Ensino Superior (Ifes) quanto os esforços de instituições privadas, notadamente as Instituições Comunitárias de Ensino Superior (ICES). No caso dessas últimas, foram preponderantes os esforços de pesquisadores e o apoio institucional para a abertura, a permanência e o aperfeiçoamento na direção das diretrizes de avaliação do SNPG. No caso do Centro-Oeste e dos programas que foram constituídos ao longo desses anos, a implementação dos cursos configurou desafios relativos à descentralização geográfica e do tipo de instituição, considerando que a maior parte dos programas está em Ifes (Costa \& Yamamoto, 2016).

Paralelamente ao processo de implantação dos cursos, os critérios de avaliação impelem à produção e, nesse sentido, a contínua articulação com as agências de fomento se coloca como um pilar fundamental, tanto para a formação quanto para a produção de conhecimentos, mas historicamente também se concentrou, ao longo dos anos, no Sudeste (Hutz, Rocha, Spink, \& Menandro, 2010).

\section{Breve histórico do Programa de Pós-graduação e Psicologia (PSSP)}

O Programa de Pós-Graduação em Psicologia da PUC-Goiás teve início em 1999, com o curso de Mestrado em Psicologia. O contexto da educação superior em que o PSSP é fundado caracterizava-se por grande carência de formação qualificada na região Centro-Oeste, uma vez que os cursos de graduação em psicologias da PUC-Goiás e da UCDB, em Campo Grande, eram os únicos da região Centro-Oeste, excetuando-se os cursos da UnB e do Uniceub, localizados no Distrito Federal.

Na criação do programa, a configuração estabeleceu três áreas de concentração: Processos Básicos, Processos Clínicos e Processos Psicossociais; visando qualificar profissionais com um perfil mais especializado. Os anos subsequentes foram marcados por um crescimento vertiginoso da Educação Superior no CentroOeste, com a fundação de vários cursos de graduação em Psicologia e áreas afins, movimento identificado por Lisboa e Barbosa (2009). Em decorrência dessa expansão, houve um aumento significativo na demanda por 
pós-graduação stricto sensu, sendo necessário expandir o PSSP, expansão que resulta na criação do doutorado em psicologia após oito anos de seu início, em 2007. Esse processo e a autoavaliação possibilitaram verificar que o perfil regional dos discentes denotava carência de uma formação mais ampla em psicologia, o que levou a uma adequação do programa e à confluência para uma área de concentração com três linhas de pesquisa: Análise e Evolução do Comportamento; Psicopatologia Clínica e da Saúde; e Psicologia Social, do Trabalho e das Organizações, oferecendo uma formação geral em termos teórico-metodológicos (http://sites.pucgoias.edu. br/pos-graduacao/mestrado-doutorado-psicologia).

$\mathrm{Na}$ perspectiva de reavaliação contínua do programa, em 2018 foi realizada uma nova estrutura para o PSSP, organizada em três linhas de pesquisa, a saber: Análise do Comportamento; Saúde: Processos, Avaliação e Intervenção; e Processos Psicossociais. Essa nova estrutura tem permitido mais integração entre as linhas, com disciplinas comuns e específicas, possibilitando ao discente inserir-se em disciplinas e projetos das demais linhas.

A ampliação do programa levou a contínuas reestruturações curriculares, atualizações das linhas de pesquisa e estabelecimento de redes de intercâmbio em pesquisa como forma de consolidar e fortalecer 0 programa. Assim, o programa apresenta resultados de sua qualificação com o aumento da produção docente e discente em periódicos Qualis de origens internacionais e nacionais, organização de livros e capítulos, além da inserção de alguns de seus docentes no programa de bolsa de produtividade do CNPq. O PSSP tem como perspectiva continuar o processo de crescimento qualificado de sua produção acadêmica, com vistas a fortalecer seus estudos e destacar-se como formação qualificada na região.

\section{Método}

\section{Amostra e Procedimentos}

As produções foram selecionadas através do critério de amostragem sistemática, respeitando os critérios de inclusão e exclusão. Os critérios de inclusão foram: ser uma dissertação/tese defendida entre 2001 e 2018, disponível no banco de dados de teses e dissertações (Sistema de Publicação Eletrônico de Teses e Dissertações TEDE), do Programa de Pós-graduação Stricto Sensu em Psicologia (PSSP) da PUC-Goiás e na Plataforma Sucupira, da Capes. Foram excluídas dissertações/teses que ainda não haviam sido publicadas na biblioteca digital e/ou entregues em definitivo na secretaria do programa.

Das 421 dissertações e 63 teses defendidas até o ano de 2018 no PSSP, selecionaram-se aleatoriamente 418 dissertações e 53 teses, das quais foram copiados os títulos e objetivos e palavras-chave, a fim de construir um corpus de dados a serem analisados.

A obtenção dos dados de produção e financiamento foi feita de forma independente por dois pesquisadores. Para a coleta de dados sobre a produção foram acessados os currículos lattes dos docentes pertencentes ao programa em cada um dos anos. A seleção contabilizou artigos publicados em periódicos, livros e capítulos de livros e foi realizada a limpeza para retirada de publicações repetidas em função de coautoria entre membros do programa. Para a coleta de dados de financiamento, foram levantadas as quantidades de bolsas de mestrado, doutorado, produtividade em pesquisa, pós-doutorado das diferentes agências financiadoras e aplicados valores correntes, equivalentes aos valores pagos em abril de 2019. Paralelamente, para os anos a partir de 2010, foram levantados os financiamentos obtidos para projetos de pesquisa individuais e conjuntos, sendo acessados todos os financiamentos efetivamente aprovados e implementados pelas diferentes agências (Capes, CNPq, Finep e Fapeg). Para projetos institucionais, foram registrados apenas os valores referentes às demandas atendidas do Programa. Para projetos em cooperação, o montante foi dividido de forma igualitária entre as instituições pertencentes.

\section{Análise de Dados}

As análises estatísticas (distribuição de normalidade e correlação) da produção científica dos docentes do PSSP e de recursos financeiros foram realizadas por meio do Pacote Estatístico SPSS para Windows versão 22.0.

Para análise de conteúdo dos resumos de teses e dissertações foi utilizado o software Iramuteq versão 0.7 alpha 2 (Interface de $R$ pour les Analyses Multidimensionnelles de Textes et de Questionnaires). As análises foram feitas através de nuvens de palavras e análise de similitudes. Assim, os resultados serão apresentados em um emaranhado de palavras, no qual as com mais relevância aparecem em destaque.

\section{Resultados}

Para responder o objetivo, os dados foram organizados em dois eixos: análise das correlações entre produção docente e investimento externo, de acordo com os 
recursos financeiros obtidos em formato de financiamento de projetos e bolsa; e análise do perfil da produção científica pela identificação dos eixos temáticos centrais das teses e dissertações produzidas nas duas últimas décadas.

\section{Análise das Correlações entre Produção Científica dos} Docentes e a Obtenção de Recursos Financeiros

A Figura 1 mostra a variação do 1) número total de produtos científicos (PRD [-] - artigos, capítulos de livros e livros), bem como a variação da 2) quantidade total de recursos financeiros obtidos pelo PSSP (FNC [O] em milhões de Reais - bolsas de estudo [M, D e PD], bolsas de pesquisa [PQ], recursos de projetos e programas específicos e recursos obtidos em editais de fomento ao programa como um todo) divididos pelo número de docentes ativos no PSSP no período de 20 anos, compreendido entre os anos de 1999 (início do programa) e 2018.



Figura 1. Comparação entre Investimento Financeiro (Fnc) em Milhões e Número de Publicações (Prd) por Docente Permanente, de 1999 a 2018.

Observa-se que os valores de ambas as variáveis aumentaram ao longo do tempo até os anos de 2013 (FNC) e 2014 (PRD). A taxa porcentual média anual de aumento do PRD por docente permanente foi de $22,06 \%$ e a taxa porcentual média anual de aumento da FNC por docente permanente foi $32,80 \%$. A comparação entre esses dois valores médios com a técnica $Z$, de Kolmogorov-Smirnov, mostrou ser a diferença entre eles não significativa estatisticamente $(Z=1,78 ; p .<0,00)$. Adicionalmente, a análise de correlação de Pearson entre os valores PRD e FNC por docente permanente mostrou correlação moderada entre as variáveis ( $r=0,65 ; p .<0,018$, bicaudado).

Não obstante a não existência de diferença entre as taxas porcentuais médias de aumento por docente e a correlação moderada verificada entre elas, observa-se que o curso de variação das taxas diferiu ao longo do tempo. Enquanto o aumento do PRD ocorreu gradual e continuamente, à exceção de três picos em 2003, 2004 e 2013, variação da FNC se deu em três momentos distintos quanto à taxa: um primeiro período com variações sem tendência crescente ou decrescente, entre 1999 e 2010; um segundo momento com crescimento acelerado, a partir de 2010 até 2014; e o terceiro, de 2014 a 2018 com uma queda inicial e variações sem tendência clara nos últimos anos.

Tomado o ano de 2011 como referência, as distribuições totais de ambas as variáveis foram subdivididas em duas distribuições parciais: PRD_1999-2010, PRD_2011-2018, FNC_1999-2010 e FNC_2011-2018. A taxa porcentual média anual de aumento do ntacmPRD_1999-2010 foi de 4,48 ( $D P=2,14)$, enquanto 
a do PRD_2011-2018 foi de 6,55 (DP = 1,11). A taxa porcentual média anual de aumento da FNC_1999-2010 foi de 1,01 ( $D P=0,32)$, enquanto a da FNC_2011-2018 foi de 12,55 ( $D P=5,26)$. As taxas porcentuais médias anuais das distribuições parciais de ambas as variáveis foram interpretadas como independentes e comparadas com o uso do teste de iterações de Wald-Wolfowitz (estatística $Z_{w-w}$ ). Desse modo, a comparação entre as taxas médias anuais do PRD_1999-2010 e do PRD_2011-2018 mostrou não haver diferença estatística significativa entre elas $\left(Z_{w-w}=-1,70\right.$; p. > 0,05). O mesmo não se passou com a comparação entre as taxas médias anuais da FNC_1999-2010 e da FNC_2011-2018, que provou haver diferença estatística significativa entre elas $\left(Z_{w-w}=-3,74 ; p .<0,00\right)$.

Adicionalmente, análises de correlação ( $r$ de Pearson) entre os valores do PRD_1999-2010 e da FNC_1999-2010, e do PRD_2011-2018 e da FNC_20112018 apresentou correlações de $0,82(F=15,98$, $p=0,004)$ e $0,45(F=1,54, p=0,26)$, respectivamente, entre os valores das variáveis nas distribuições parciais.

\section{Perfil da produção científica}

Abaixo é apresentada a nuvem de palavras referente às dissertações e, posteriormente, às teses defendidas. Para avaliar os dois grupos foi utilizada a técnica nuvem de palavras, que tem como objetivo inferir a relevância de certas palavras, e a análise de similitudes, que indica a conexão entre as palavras, auxiliando na identificação da estrutura do conteúdo de um corpus textual. As palavras "objetivos" e "estudo" fazem parte da descrição do objetivo do trabalho, por isso foram retiradas das análises dos temas pesquisados nas dissertações e teses.

O corpus geral das dissertações foi constituído por 418 textos, separados em 592 seguimentos de texto (ST). Emergiram 19500 ocorrências (palavras, formas ou vocábulos), sendo 3150 formas distintas e 1447 com uma única ocorrência. O corpus geral das teses foi constituído por 53 textos, separados em 102 seguimentos de texto (ST). Daí emergiram 3394 ocorrências, sendo 989 formas distintas e 575 com única ocorrência.

\section{Análise das Dissertações}

Como demonstrado na Figura 2, no que se refere às dissertações as palavras "comportamento" e "social" se destacam, demonstrando uma produção em duas áreas distintas da psicologia, de um lado a análise comportamental e de outro a psicologia social. Isto fica mais evidente na Figura 3, pois se percebe a distancia entre os dois temas que compõem ramos diferentes da árvore resultante da análise de similitudes.

No conjunto de estudos reunidos em "comportamento", observam-se expressões que indicam um domínio da abordagem da análise do comportamento. Essa proximidade demostra relação com as palavras "análise", "efeito", "avaliar", "resposta", "esquizofrenia" e "estímulo".

Em seguida pode-se perceber um conjunto de palavras que remetem a fases do desenvolvimento (criança e adolescente) próximas da palavra relação. Ao observar a Figura 3, percebe-se que essas fases do desenvolvimento se associam às abordagens comportamentais sendo portanto distantes das perspectivas sociais. No entanto, nos estudos sobre infância e adolescência as palavras próximas não parecem indicar, prioritariamente, relação com as abordagens comportamentalistas. Palavras como "sentido", "significado" e "violência" parecem indicar os temas estudados com esses públicos.

Próximo à palavra criança, há outras palavras que demonstram o interesse pelas aquisições do desenvolvimento humano, físico e sexual. Em torno da palavra adolescente, aparecem as palavras sentido e significado fenomenológico que se referem provavelmente ao sentido existencial da vida que é complementado pelos termos autor e violência. Desta forma fica evidenciado que no que tange a infância estuda-se o desenvolvimento físico e sexual enquanto que no que tange a adolescência estuda-se o sentido da vida, as violências sofridas e seus autores.



Figura 2. Nuvem de Palavras Referente às Dissertações Defendidas no Programa entre os anos de 2001 (Primeira Defesa de Ingressantes de 1999) e 2018. 


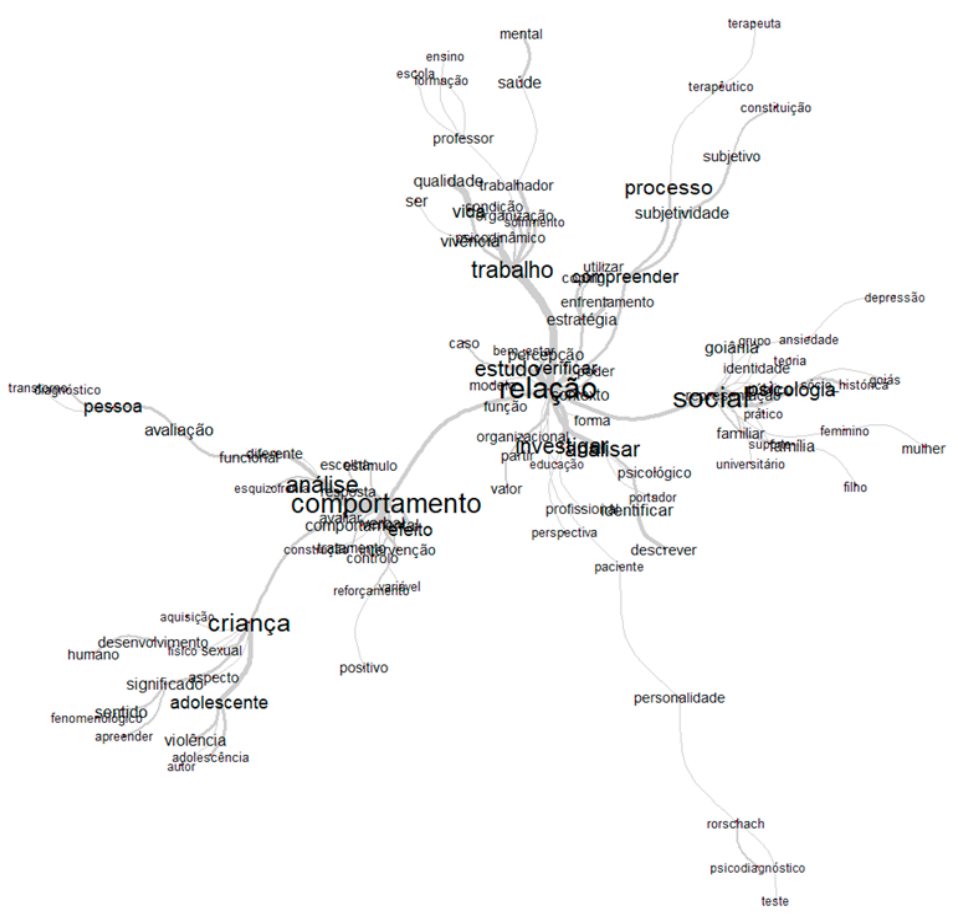

Figura 3. Gráfico de Similitude das Palavras Referente às Dissertações Defendidas no Programa entre os Anos de 2001 (Primeira Defesa de Ingressantes de 1999) e 2018.

$\mathrm{Na}$ Figura 3 fica evidenciado que esse interesse por fases do desenvolvimento é substituído pelo interesse na família, no papel de filho ou de universitário, no feminino e na mulher quando considera-se o conjunto ligado à palavra social. Evidencia-se que o interesse é pelos grupos e identidades, surgindo referências históricas e geográficas como a cidade de Goiânia e o estado de Goiás, e referências a males da sociedade como a depressão e ansiedade.

Sobre a palavra "relação", observa-se que ela se apresenta como o centro da análise de similitude, ligando todos os ramos da árvore junto com outras palavras como verificar, investigar e estudo. Ela se liga, além dos dois ramos principais (comportamento e social) que acabamos de descrever, com dois outros ramos nos quais as principais palavras são "trabalho" que tem próximo de si as expressões "vivência" e "psicodinâmico"; e "processo" que está ligada a "subjetividade". Esses dois ramos parecem indicar estudos sobre a psicodinâmica do trabalho e sobre a subjetividade termo que indica uma perspectiva marxista.

Em resumo, os temas das dissertações de mestrado do PSSP poderiam ser divididos em quatro categorias: 1-Estudos que enfatizam o comportamento com ênfase na infância e adolescência; 2-Estudos de psicologia social sobre grupos, identidades, papéis sociais e gênero numa perspectiva local e regional; 3-Estudos relacionados ao trabalho numa abordagem psicodinâmica e; 4- Estudos que focalizam a subjetividade.

Para auxiliar na compreensão das expressões presentes na análise de similitude, a Tabela 1 apresenta a frequência das palavras que surgiram mais de 30 vezes nos ST. A palavra "social" vem com 187 ocorrências, seguida de "comportamento", com 177, "trabalho", com 160 e "relação" com 146. "Criança" teve 143 e as palavras "análise" e "adolescente" apareceram em 95 ocorrências cada uma.

Tabela 1. Frequência (F) de Formas Ativas que Apareceram a Partir de 31 Vezes no ST das Dissertações

\begin{tabular}{lcll}
\hline Formas Ativas & F & Formas Ativas & F \\
\hline comportamento & 177 & contexto & 49 \\
trabalho & 160 & compreender & 49 \\
relação & 146 & trabalhador & 48 \\
criança & 143 & goiânia & 48 \\
análise & 95 & subjetivo & 46 \\
adolescente & 95 & funcional & 46 \\
vida & 83 & família & 46 \\
processo & 82 & estratégia & 45 \\
violência & 78 & sexual & 42 \\
estudo & 75 & intervenção & 41 \\
investigar & 73 & identificar & 41 \\
psicologia & 72 & público & 40 \\
analisar & 72 & familiar & 40 \\
& & & Continua...
\end{tabular}


Periférico e comunitário: características e desafios de um programa de pós-graduação em Psicologia no Centro-Oeste

Tabela 1. Continuação

\begin{tabular}{llll}
\hline Formas Ativas & F & Formas Ativas & F \\
\hline qualidade & 70 & psicológico & 39 \\
sentido & 64 & professor & 39 \\
verbal & 62 & escolha & 38 \\
pessoa & 62 & desenvolvimento & 38 \\
subjetividade & 61 & comportamental & 38 \\
avaliação & 58 & personalidade & 36 \\
saúde & 56 & mulher & 35 \\
representação & 56 & bem_estar & 33 \\
efeito & 55 & tratamento & 32 \\
verificar & 54 & modelo & 32 \\
vivência & 53 & estímulo & 32 \\
percepção & 53 & contra & 32 \\
coping & 52 & resposta & 31 \\
organizacional & 50 & psicodinâmico & 31 \\
significado & 49 & poder & 31 \\
valor & 39 & & \\
\hline
\end{tabular}

\section{Análise das Teses}

$\mathrm{Na}$ Figura 4, observa-se que se destacam mais as palavras "psicodinâmico", "relação" e "social". Em seguida aparecem as palavras "comportamento" e "representação". No entanto, quando analisamos a árvore de similitude percebemos que essas palavras se organizam em quatro subgrupos de palavras que demonstram a divisão dos estudos em conjuntos: 1- comportamento; 2- psicodinâmica; 3-relação e; 4- representação.

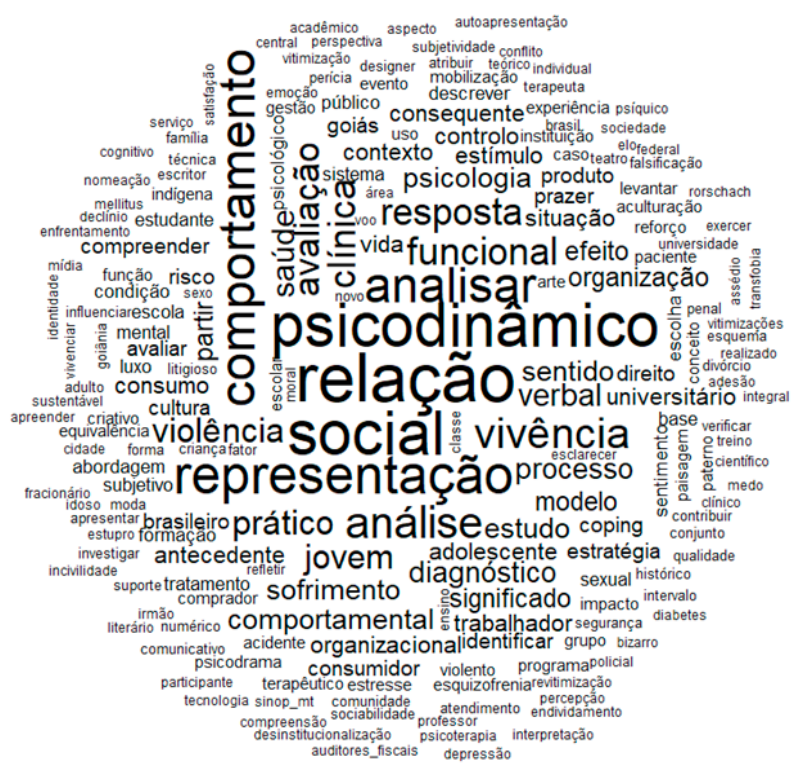

Figura 4. Nuvem de Palavras Referente às Teses Defendidas no Programa de 2010 a 2018.

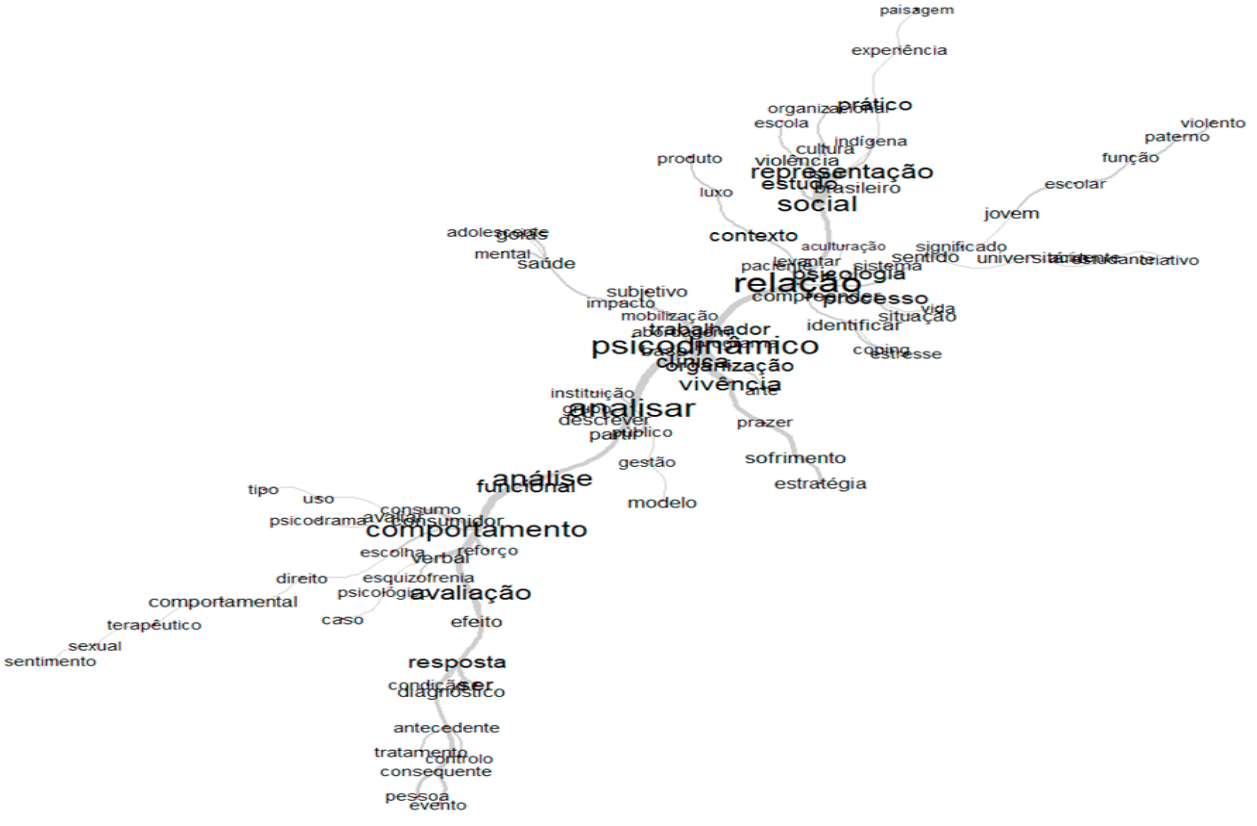

Figura 5. Gráfico de Similitude de Palavras Referente às Teses Defendidas no Programa de 2010 a 2018. 
O primeiro grupo identifica estudos do comportamento com temas relacionados a clínica, avaliação e diagnóstico; aos conceitos da análise do comportamento como efeito e resposta; e também ao comportamento do consumo e do consumidor.

Os estudos em psicodinâmica, do segundo subgrupo, reúnem estudos em "saúde mental" e "adolescentes" e de outro lado estudos sobre "clínica", "vivência", "prazer" e "sofrimento". Bem próximo à palavra psicodinâmica fica evidente que todas essas palavras se referem a dois contextos que são a clínica e o trabalho.

As palavras do terceiro subgrupo, representado pelo termo "relação parecer dizer" sobre as metodologias dos estudos, assim como um subgrupo menor que se forma em torno da palavra "analisar". A temática relacionada a "psicodinâmica" está entre os dois subgrupos, enquanto que a temática "os estudos de comportamento", está mais próxima a "analisar", e os estudos "sociais" estão mais próximos de "relação".

E, por fim, o subgrupo em torno da palavra representação que se associa ao termo social e trata da violência e da cultura do brasileiro, do jovem e do escolar.

Em resumo, nas teses do PSSP também fica evidente a dicotomia entre estudos que focalizam o comportamento e outros que concentram seus esforços no aspecto social. No entanto, entre eles, aparece um terceiro grupo de temas de abordagem psicodinâmica.

A Tabela 2 também apresenta as formas ativas mais frequentes nos ST das teses. As palavras de maior ocorrência são "relação" com 29 ocorrências, "social" e "psicodinâmico", com 26 ocorrências cada uma, seguido por "representação" com 22 ocorrências.

Tabela 2. Frequência (F) de Formas Ativas que Apareceram a Partir de 10 Vezes no ST das Teses

\begin{tabular}{llll}
\hline \multicolumn{1}{c}{ Formas ativas } & F & Formas ativas & F \\
\hline relação & 29 & violência & 13 \\
social & 26 & prático & 13 \\
psicodinâmico & 26 & jovem & 13 \\
representação & 22 & verbal & 12 \\
comportamento & 21 & saúde & 12 \\
analisar & 20 & processo & 11 \\
análise & 18 & diagnóstico & 11 \\
vivência & 17 & sofrimento & 10 \\
resposta & 15 & sentido & 10 \\
funcional & 15 & psicologia & 10 \\
clínica & 15 & estudo & 10 \\
avaliação & 14 & comportamental & 10 \\
\hline
\end{tabular}

\section{Discussão}

Este trabalho objetivou avaliar a associação entre investimento externo (bolsas e recursos advindos de projetos financiados) e produção docente e analisar o perfil da produção científica discente, pela identificação dos eixos temáticos centrais das teses e dissertações produzidas nas duas últimas décadas. Para tal, foram feitas duas análises documentais distintas, sendo uma delas focada na relação entre produção docente e obtenção de recursos financeiros advindos de instituições de fomento à pesquisa em Goiás (Fapeg) e no Brasil (Capes, Finep e CNPq) e a outra na análise dos eixos temáticos das teses e dissertações produzidas no programa.

As produções científicas dos docentes vinculados ao PSSP apresentam um aumento contínuo e gradual desde a criação do programa, em 1999, até o ano de 2018. À medida que o programa foi se consolidando, houve constância nas publicações docentes, proporcional às defesas de dissertações e teses. Em 2007, com o aumento da nota do programa na Capes, foi possível instituir o doutorado, o que caracterizou um marco para o PSSP. A partir de então, houve um discreto aumento na obtenção de recursos externos, em virtude da inserção de docentes em redes de intercâmbio em pesquisas nacionais e internacionais, assim como pela obtenção de recursos para o desenvolvimento de pesquisas junto às agências de fomento. Esse movimento explicita um dos grandes desafios da interiorização e da consolidação de um programa nos moldes e temporalmente localizado como o PSSP, diferenciando-se do processo de consolidação dos programas centrais. Para estes, o processo de consolidação ocorreu concomitantemente ao aporte de recursos. Para os programas interiorizados, a consolidação teve uma função de impulsionamento de recursos posteriormente a esse processo.

Em que pese as regras e exigências estabelecidas pelo Sistema Nacional de Pós-Graduação (SNPG) influenciarem o aumento da produção no PSSP, assim como ocorreu nos demais programas de pós-graduação no Brasil, houve um empenho maciço dos docentes desse programa pela qualificação da produção científica e em fazer frente aos editais de incentivo à pesquisa. $\mathrm{O}$ aumento do financiamento, portanto, se associa pela qualidade dos projetos desenvolvidos pelos docentes da PUC-Goiás e pelas políticas públicas dos governos Federal e Estadual, na esteira dos incentivos às atividades estabelecidas de pesquisa científica e de formação de recursos humanos na região Centro Oeste e no país como um todo. 
Os dados levantados demonstram ser o ano de 2012 outro marco para o PSSP, evidenciado no aumento vertiginoso e cumulativo de recursos externos recebidos das agências de fomento. Esse aumento pode ter relação com a produção científica docente observada nos anos anteriores, o que o qualificou para competir de maneira mais direta nos editais. Os resultados deste estudo evidenciam que, embora o financiamento seja uma variável importante para viabilizar a produção, não é, certamente, a única, podendo ser melhor interpretada como variável estruturante, pré-requisito para a produção. Neste sentido, o financiamento pode ser analisado como variável meio, ao possibilitar a criação de mecanismos que, estes sim, mais provavelmente repercutirão em aumento da produção, tais como, por exemplo, a criação de redes de colaboração entre pesquisadores, o acesso a contextos mais avançados de pesquisa (via estágios pós-doutorais) e a atualização de recursos tecnológicos para a pesquisa.

O crescimento do programa com a inclusão do doutorado, em 2007, o aumento constante e regular da produção científica docente, assim como a inserção de seus egressos no mercado de trabalho dão consistência ao projeto de interiorização da pós-graduação e de redirecionamento de recursos, conforme defendem Tourinho e Bastos (2010). Num país de dimensões territoriais enormes como o Brasil, torna-se fundamental a efetivação de políticas de interiorização da Educação no âmbito da pós-graduação, dando acesso à formação qualificada fora do eixo Sul e Sudeste.

O perfil da produção científica discente pode ser evidenciado pelos eixos temáticos centrais das teses e dissertações produzidas nas duas últimas décadas. Os dados demonstram haver dois eixos temáticos principais, sendo eles vinculados tanto à perspectiva social quanto à individual de concepção do sujeito. Ao destacar esses eixos vinculados às palavras "social" e "comportamento", os dados refletem a dicotomia presente na psicologia desde os seus primórdios. Por um lado, a palavra "comportamento", utilizada amplamente pelas correntes comportamentalistas, denota uma leitura mais individualista do fenômeno humano. Por outro, a palavra "social" representa uma gama de autores e obras da psicologia que privilegiam os fatores sociais, culturais e econômicos. Apesar de não terem sido evidenciados entre os eixos principais, as perspectivas no campo da subjetividade e da psicodinâmica também se fazem presentes. Essa dicotomia pode ser explicada pela própria estrutura do PSSP, dividido em três linhas de pesquisa, a saber: Análise do Comportamento; Saúde: Processos, Avaliação e Intervenção; e Processos Psicossociais.
A linha Análise do Comportamento "[...] desenvolve pesquisas básicas e aplicadas referentes a processos comportamentais verbais e não verbais em contextos experimentais, clínicos e comunitários". A linha Saúde: Processos, Avaliação e Intervenção, apesar de não apresentar, em seu título, as palavras "social" e "comportamento", tem como propósito "desenvolver pesquisas relacionadas à avaliação e intervenção em aspectos psicológicos do bem-estar, prazer e sofrimento, sob perspectivas quantitativas ou qualitativas, nos contextos clínico, hospitalar, organizacional, do trabalho e situações sociais". Portanto, se caracteriza como uma linha de pesquisa que engloba tanto os aspectos individuais como grupais e sociais dos fenômenos psicológicos. Nessa esteira de articulações, a Linha Processos Psicossociais [...] realiza pesquisas e intervenções em processos sociais, objetivando a produção de conhecimento sobre fenômenos sociais complexos, abordados a partir do reconhecimento das diferentes modalidades do pensamento social e o estudo das relações entre o indivíduo e a sociedade, e dos processos intra e intergrupais característicos da dinâmica destas relações" (site do PSSP).

Tomadas em conjunto, as linhas de pesquisa desenvolvidas no programa ancoram uma perspectiva abrangente da psicologia, vindo ao encontro do que defendem Camino, Torres, Lima e Pereira (2013) em termos dos aspectos que reafirmam uma relação estreita entre a psicologia individual e a psicologia grupal, englobando tanto os processos de individuação como aqueles que privilegiam os estudos intra e intergrupais. Além disso, vale lembrar que o aumento do investimento em pesquisa em regiões fora do eixo sul sudeste também possibilita o desenvolvimento de estudos que busquem responder a problemas vivenciados nesta realidade específica.

\section{Conclusão}

Tomadas em conjunto as análises de financiamento e produção por docente permanente e das palavras nas dissertações e teses verifica-se que ao longo do período temáticas consistentes foram desenvolvidas, caracterizando uma maior maturidade do programa expressa em uma identidade voltada para estudos direcionados para a compreensão do comportamento e para demandas sociais, nas vertentes da psicologia que o programa atua. Isso é ilustrado pelo aumento nas taxas da produção e financiamento por docente e a manutenção das temáticas que envolvem necessidades regionais e nacionais, 
tanto em termos de produção de conhecimentos básicos quanto aquelas voltadas para o fornecimento de procedimentos e técnicas aplicados embasados cientificamente. Essa dinâmica, como fruto de um processo de expansão e interiorização da pós-graduação em psicologia tem contribuído para a consolidação da identidade do programa em consonância com sua repercussão em termos de formação de pessoal e disseminação de conhecimentos qualificados que atendam ao mesmo tempo os critérios dos órgãos de controle e avaliação e essas demandas.

Em suma, este trabalho demonstra haver associação entre a produção científica dos docentes e os recursos financeiros recebidos de instituições de fomento à pesquisa. Certamente, os efeitos de financiamentos aparecem também no longo prazo, o que justifica um acompanhamento longitudinal para avaliar os efeitos desse crescimento sobre a quantidade e qualidade da produção nos próximos anos. Além disso, demonstrou que os eixos temáticos dos trabalhos defendidos no programa estão alinhados com as linhas de pesquisa nele desenvolvidas. Em conjunto, esses dados revelam o esforço que um programa de uma instituição periférica e comunitária tem feito para atender às demandas da Capes.

O estudo apresenta algumas limitações a serem consideradas em pesquisas posteriores, entre elas destaca-se a necessidade de aprofundar-se sobre os aspectos do contexto social e organizacional que impactam na produção docente, das estratégias que têm sido desenvolvidas nos últimos anos para a qualificação da produção, assim como analisar as variáveis econômicas e políticas que podem impactar as instituições de natureza comunitária em termos de número de matrículas, incentivo à pesquisa, bolsas de estudo, e outros aportes financeiros.

Portanto, conclui-se que o PSSP é um programa que vem contribuindo significativamente para a interiorização das pós-graduações em psicologia no Brasil, construindo pesquisas que possuem uma coerência temática e que expressam os interesses não apenas regionais, mas da psicologia enquanto ciência mundial. Destaca-se ainda que os financiamentos de pesquisa foram expressivamente importantes para o crescimento da produção científica demonstrando que os investimentos direcionados a programas fora dos grandes centros foram efetivamente direcionados para seus fins alcançando os resultados esperados. Desta forma, espera-se que as políticas de pesquisa de incentivo à interiorização das pós-graduações continuem a fim de manter e fazer crescer programas como o PSSP.

\section{Referências}

Bastos, A. V. B., Gondim, S. M. G., \& Rodrigues, A. C. A. (2010). Uma categoria profissional em expansão: quantos somos e onde estamos? In A. V. B. Bastos \& S. M. G. Gondim (Orgs.), O trabalho do psicólogo no Brasil (pp. 32-44). Porto Alegre: Artmed.

Camino, L., Torres, A. R. R., Lima, M. E. O., \& Pereira, M. E. (2013). Psicologia social: temas e teorias ( $2^{\underline{a}}$ ed. ampliada e revisada). Brasília: Technopolitik.

Coordenação de Aperfeiçoamento de Pessoal de Nível Superior - CAPES. (2010). Plano Nacional de Pós-Graduação [PNPG] 2011-2020 (Vol. 1). Brasília, DF: CAPES. Recuperado de https://www.capes.gov.br/ images/stories/download/Livros-PNPG-Volume-I-Mont.pdf

Coordenação de Aperfeiçoamento de Pessoal de Nível Superior CAPES. (2017). Relatório de Avaliação Quadrienal 2017 - Psicologia. Brasília, DF: CAPES. Recuperado de https://capes.gov.br/images/ documentos/Relatorios_quadrienal_2017/20122017-Psicologia_ relatorio-de-avaliacao-2017_final.pdf

Costa, A. L. F., \& Yamamoto, O. H. (2016). Políticas sociais na pós-graduação stricto sensu de Psicologia. Estudos de Psicologia, 21(4), 456-467. doi: 10.5935/1678-4669.20160044

Delmondes, G. F. S. (2018). Memória e história dos primeiros anos do curso de graduação em psicologia fadafi/fucmt (1974-1980) (Dissertação de mestrado, Universidade Católica Dom Bosco, Campo Grande, MS). Recuperado de https://site.ucdb.br/public/ md-dissertacoes/1023280-final-compressed-5.pdf

Hutz, C. L., Rocha, M. L., Spink, M. J., \& Menandro, P. R. M. (2010). Perfil, avaliação e metas da produção intelectual dos programas de pós-graduação em Psicologia. Psicologia: Reflexão e Crítica, 23(supl. 1), 25-34. doi: 10.1590/S0102-79722010000400004

Lisboa, F. S., \& Barbosa, A. J. G. (2009). Formação em Psicologia no Brasil: um perfil dos cursos de graduação. Psicologia: Ciência e Profissão, 29(4), 718-737. doi: 10.1590/S1414-98932009000400006

Macedo, J. P., \& Dimenstein, M. (2011). Expansão e interiorização da Psicologia: reorganização dos saberes e poderes na atualidade. Psicologia: Ciência e Profissão, 31(2), 296-313. doi: 10.1590/ S1414-98932011000200008

Morosini, M., \& Franco, M. A. D. P. (2006). Universidades comunitárias e sustentabilidade: desafio em tempos de globalização. Educar em Revista, 28, 55-70. doi: 10.1590/S0104-40602006000200005

Steiner, J. E. (2005). Qualidade e diversidade institucional na pós-graduação brasileira. Estudos Avançados, 19(54), 341-365. doi: 10.1590/S0103-40142005000200019

Tourinho, E. Z., \& Bastos, A. V. B. (2010). Desafios da pós-graduação em Psicologia no Brasil. Psicologia: Reflexão e Crítica, 23(Suppl. 1), 35-46. doi: 10.1590/S0102-79722010000400005

Veiga, F. H. (2014). Internationalization of teaching and research in Psychology and Education: Portugal-Brazil demands. Estudos de Psicologia, 31(2), 149-158. doi: 10.1590/0103-166X2014000200001

Venanzi, D., \& Sandano, W. (2014). Universidades comunitárias: as características na prestação de serviços públicos. Revista Temas em Educação, 23(2), 140-155. Recuperado de http://www.periodicos. ufpb.br/ojs/index.php/rteo/article/view/20501/12569 
1. Dado calculado a partir das informações disponíveis na Plataforma Sucupira, da Capes, em 30/05/2019. Recuperado de https://sucupira.capes. gov.br/sucupira/public/consultas/coleta/programa/quantitativos/quantitativoRegiao.jsf

Daniela Sacramento Zanini, Doutora em Psicologia Clínica e da Saúde pela Universidad de Barcelona - UB, Espanha, Pós-Doutora pela Universidad de Barcelona - UB, Espanha (2008) e pela Universidade do Porto (Portugal) (2020), é Professora Adjunta da Pontifícia Universidade Católica de Goiás - PUC/Goiás. Endereço para correspondência: Rua $9 \mathrm{n}^{\circ} 769$ apt 902, Setor Oeste, GoiâniaGoiás. Telefones: (62) 999841664 e (62) 39461116. Email: dazanini@yahoo.com

Margareth Regina Gomes Verissimo Faria, Doutora em Psicologia pela Pontifícia Universidade Católica de Goiás/Psicologia -

PUC/Goiás, Pós Doutora em Psicologia pela Universidade de São Paulo - USP/Ribeirão Preto, é Professora Horista Assistente da Pontifícia Universidade Católica de Goiás - PUC/Goiás, é Professora Adjunta do Centro Universitário de Anápolis/GO - UniEvangélica. Email: margarethverissimo@gmail.com

Lauro Eugênio Guimarães Nalini, Doutor em Psicologia pela Universidade de Brasília - UnB, é Professor Adjunto da

Pontifícia Universidade Católica de Goiás - PUC/Goiás. Email: nalini00@gmail.com

Cristiano Coelho, Doutor em Psicologia pela Universidade de Brasília- UnB, é Professor Adjunto da Pontifícia Universidade Católica de Goiás - PUC/Goiás.

Email: cristiano@pucgoias.edu.br

Helenides Mendonça, Doutora em Psicologia pela Universidade de Brasília - UnB,

Pós-Doutora pelo Instituto Universitário de Lisboa, é Professora Titular da Pontifícia Universidade de Lisboa. Email: helenides@gmail.com

Sebastião Benicio da Costa Neto, Doutor em Psicologia pela Universidade de Brasília - UnB, Pós-Doutor em Psicologia pela Universidade Federal do Rio Grande do Sul - UFRGS, Pós-Doutor em Psicologia pela Escola de Educação/Faculdade de Psicologia

da Universidade do Minho - UMINHO, é Professor Adjunto da Pontifícia Universidade Católica de Goiás - PUC/Goiás, e Psicólogo Hospitalar da Universidade Federal de Goiás (HC/UFG/EBSERH). Email: sebastiaobenicio@gmail.com

Iorhana Almeida Fernandes, Doutoranda em Psicologia pela Pontifícia Universidade Católica de Goiás - PUC/GO, é Professora no Centro Universitário de Anápolis - UniEVANGÉLICA. Email: iorhanafernandes@hotmail.com

Lila Maria Spadoni Lemes, Doutora em Psicologia pela Université Paris Descartes (Paris 5- França), é Professora assistente da Pontifícia Universidade Católica de Goiás - PUC/GO. Email: lilaspadoni@gmail.com

Recebido em 11.jun.19 Revisado em 17.out.19 Aceito em 31.dez.19 